An Introduction to Modern Organic Analysis By Sidney Siggia and Hans J. Stolten. Pp. vii +250. (New York : Interscience Publishers, Inc.; London: Interscience Publishers, Ltd., 1956.) 4.50 dollars.

M OST modern books on analysis are necessarily 1 specialized. They deal with only one type of approach to the problem and describe in detail the application of one technique or group of associated techniques. This excellent book, on the other hand, surveys the whole field of organic analysis and introduces the reader to the general strategy of the investigation of organic materials, discussing a few perticular examples by way of illustration. Full working details are not, in general, provided, but at the end of each chapter one or two standard works and references are listed, from which the reader may obtain the necessary tactical details.

In successive chapters the principles and application of various methods of attack are described: elementary analysis ; the determination of functional groups; potentiometric titration; absorption spectroscopy in the visible, ultra-violet and infra-red regions; polarography and X-ray diffraction. Analytical uses of freezing-point, refractive index and density are discussed. Various methods for separating mixtures are reviewed, including chromatography and ion-exchange, and brief mention is made of a number of more specialized devices. Although most of these subjects are dealt with very concisely, the sections on group analysis and on absorption spectrometry are particularly well done.

This volume was based on lectures to adult audiences and is not designed as a conventional student's text-book, yet it provides a most interesting and readable general introduction to the subject which should be of great value to graduates about to begin work as responsible technologists. It conveys a feeling of contact with real practical problems which is very stimulating. The book is well produced and illustrated and has an adequate index. It is remarkably good value for its moderate price.

\section{G. M. BenNETT}

\section{Encyclopedia of Chemical Reactions}

Compiled by Prof. C. A. Jacobson. Edited by Clifford A. Hampel. Vol. 6 : Samarium; Scandium; Selenium; Silicon; Silver; Sodium. Pp. vii +438. (New York: Reinhold Publishing Corporation; London: Chapman and Hall, Ltd., 1956.) 100s. net.

THIS encyclopedia of chemical reactions is based upon reactions abstracted by some 100 abstractors from technical and scientific periodicals. The abstractors worked under the direction of Dr. C. A. Jacobson, lately professor of chemistry in the University of West Virginia, who had as his target a compilation of chemical reactions involving inorganic reagents. This volume deals with reactions involving samarium, scandium, selenium, silicon, silver and sodium. The information is very concise as the following example indicates. "Electricity makes silver react with carbon dioxide, forming silver oxide and carbon monoxide. $2 \mathrm{Ag}+\mathrm{CO}_{2} \rightarrow \mathrm{Ag}_{2} \mathrm{O}+\mathrm{CO}$." The reference is W. R. Grove, Phil. Trans. Roy. Soc. (London), 142, 94; 1852. Under the heading of sodium chloride on p. 273 an abstract informs the reader "Silver nitrate is added to the solution to precipitate any soluble chloride, as is well known, as silver chloride. Sodium is used as an example and the corresponding nitrate is also formed. $\mathrm{AgNO}_{3}+$ $\mathrm{NaCl} \rightarrow \mathrm{AgCl}+\mathrm{NaNO}_{3}{ }^{\prime \prime}$. The reference is $\mathrm{W}$.
Crookes, Phil. Trans. Roy. Soc. (London), 174, 897 ; 1883. It would be unfair to leave the impression that the information is all of this vintage, but it is very doubtful whether these abstracts are worth the 100s. which is the price of this volume. The best advice for the prospective buyer is to see the volume before purchasing it.

W. WARDLAW

\section{Crystallographic Data for the Calcium Silicates}

By Dr. L. Heller and Dr. H. F. W. Taylor. Pp. vi+79. (London: H.M. Stationery Office, 1956. Published for the Department of Scientific and Industrial Research.) 10s. 6d. net.

7 HIS little book summarizes the results of a programme of research into the hydration products of cement sponsored by the Building Research Board. Almost all the known natural calcium silicates and a very wide range of artificial products were examined, and for each well-defined phase the authors give, so far as possible: X-ray powder data, optical properties, density, crystal-strueture data, natural and technical occurrence, and short details of synthesis. The data appear to be carefully collected and checked, and the book should be a very useful summary for workers dealing with cement or boiler scales or with the calcium silicate minerals. In two appendixes a classification of the hydrated calcium silicates is attempted, with notes on their dehydration and on the structure types known in the group. A third appendix lists the spacings and intensities of the three strongest lines in the X-ray powder photographs of the phases described. The worst defect of the book is the lack of an index; in its absence, workers desiring to find out what is known of such 'doubtful minerals' as reyerite (p. 28), or the meaning of such old names as alite (p. 19), will probably fail to locate the information.

M. H. HEY

Table of the Fresnel Integral to Six Decimal Places Compiled by T. Pearcey. Pp. 63. (Cambridge : At the University Press, 1956.) 12s. 6d. net.

$\mathrm{HHE}$ Fresnel integral occurs in the solutions of a large number of diffraction problems, and it also frequently arises in other problems of theoretical physics. Despite the increasing availability of electronic computors, there is still a need for good tables of many functions; for often only relatively few numerical results are required; such as would not justify the expense of programming. Again, even when a calculation has been programmed, it is useful to check this by means of a desk machine computation. The present table gives :

$$
C(u)=\int_{0}^{u} \cos \frac{\pi t^{2}}{2} \mathrm{~d} t, \quad S(u)=\int_{0}^{u} \sin \frac{\pi t^{2}}{2} \mathrm{~d} t
$$

employing as the argument $x=\pi u^{2} / 2$ - the definition of $S(u)$ on page 8 gives $x$, in error, as the upper limit. The use of $x$ as the variable in place of $u$ makes linear interpolation possible throughout most of the table, but it has the disadvantage that $x=$ $50 \cdot 00$, the final entry, corresponds to only $u=5 \cdot 64$, so that beyond this relatively small value other tables or asymptotic series must be used. Between $x=0 \cdot 00$ and 1.00 the entries are to seven places, and to six for the region $x=1 \cdot 00$ to $50 \cdot 00$. Second differences are given throughout, together with a small table to facilitate their use. The table is clearly set out and very easy to use.

\section{H. H. HopkINS}

